\title{
Effects of plant growth regulators and activated charcoal on somaclonal variations of protocorm-like bodies (PLBs) of Dendrobium Sabin Blue orchid
}

\begin{abstract}
Protocorm-like bodies (PLBs) of Dendrobium orchids are emerging as a potential source of valuable secondary metabolites. This study examined the effect of four additives namely 1-naphthaleneacetic acid (NAA), kinetin, thidiazuron (TDZ), and activated charcoal (AC) used in culture medium on genetic variability in PLBs of Dendrobium Sabin Blue. Nine (9) ISSR primers and eleven (11) DAMD primers were used to assess the genetic variability of PLBs that were subcultured over a period of two years. We confirmed that the use of kinetin in culture medium for two years resulted in the highest rate of somaclonal variation in PLBs. On the other hand, TDZ and activated charcoal registered the lowest genetic variability in PLBs. The findings of this study suggest the importance of selecting additives used in the culture medium to maintain stable genetic lines of PLBs. We recommend that the assessment of somaclonal variations should be performed for long term maintenance of tissue cultures.
\end{abstract}

Keyword: Orchid; Protocorm-like bodies; Somaclonal variations; DNA markers 